\title{
A CHECKLIST OF THE FAMILY LAUXANIIDAE (DIPTERA, LAUXANIOIDEA) IN VIETNAM
}

\author{
Hyun Suk Lee ${ }^{a^{*}}$ \\ ${ }^{a}$ The Faculty of Biology, Dalat University, Lamdong, Vietnam \\ Article history \\ Received: August 10 $0^{\text {th }}, 2016 \mid$ Received in revised form: September $20^{\text {th }}, 2016$ \\ Accepted: September $30^{\text {th }}, 2016$
}

\begin{abstract}
I reviewed the lauxaniid literatures as much as possible, and listed any lauxaniid species with collecting records in Vietnam. Vietnamese lauxaniid flies belong to two subfamilies. In this paper, I provide a taxonomic checklist of 60 nominal species under the subfamily Homoneurinae. However, this checklist is based on information of diverse literatures, not confirmation of identifications with each species. I will provide other checklist of lauxaniid species under the subfamily Lauxaniinae as soon as possible. A more comprehensive research is needed to confirm identifications of previously recorded lauxaniid species in Vietnam.
\end{abstract}

Keywords: Checklist; Diptera; Homoneurinae; Lauxaniidae; Vietnam.

\section{INTRODUCTION}

The Lauxaniidae is one of the largest Acalyptratae families. There are more than 160 genera and nearly 2,000 described species, distributed worldwide except for Antarctica (Shi, Yang, \& Gaimari, 2011). Lauxaniid flies are usually found in moist shady undergrowth, grassland, all forest types, sand dunes, mangrove swamps and even on mountain summits (Kim, 1994). Saprophagous larval stages are found in leaf litter, decaying vegetation and similar habitats (Kim, 1994).

Sasakawa (2001) conducted a comprehensive taxonomic research about the Vietnamese fauna of lauxaniid flies for the first time. This work consists of 75 species including 34 new species and 40 newly recorded species (21 species belong to the Lauxaniinae and 54 to the Homoneurinae). Before this work, only three species, Sciasmomyia meijerei Hendel (1907), Trigonometopus (Tetroxyrhina) tinctipennis de Meijere (1924) and Homoneura (Homoneura) trypetoptera (Hendel, 1908) had been recorded in Vietnam (Shewell, 1977). Sasakawa (2002, 2003) also listed many

\footnotetext{
"Corresponding author: exuknight@gmail.com
} 
Vietnamese lauxaniid species in Oriental lauxaniid fauna. Shi, Yang and Gaimari (2011) described four new species from China and Southeast Asia. In this work, three new species and other three species are recorded for the first time in Vietnam. Lee et al. (2016) provided color photographs and descriptions of four Homoneurinae species in pictorial book of Southern Vietnam.

This checklist aims at reviewing the lauxaniid literatures as many as possible, and lists any lauxaniid species with Vietnamese records. In total, 60 species under the subfamily Homoneurinae are officially listed for the Vietnamese lauxaniid fauna. However, this checklist is based on information of diverse literatures, not confirmation of identifications with each species. I will provide other checklist of lauxaniid species under the subfamily Lauxaniinae as soon as possible. This checklist will be a fundamental base for comprehensive taxonomic research of lauxaniid fauna in Vietnam.

\section{RESULTS}

\section{Genus Cestrotus Loew, 1862}

TS: Cestrotus turritus Loew, 1862.

1. Cestrotus flavoscutellatus Meijere (1910, p.142). TL: Indonesia, Java, Gunung Pantjar, nr Bogor.

Vietnamese Records:

Cestrotus flavoscutellatus; Sasakawa (2001, p.54, remarks, distribution, new to Vietnam); Sasakawa (2002, p.53, distribution); Sasakawa (2003, p.63, distribution, new to Laos); Shi, Yang and Giamari (2009, pp.43, 46, 68, in Chinese key, diagnosis, description, distribution, head, thorax and wing photo, male genitalic figure, remarks); Shi, Yang and Gaimari (2011, p.673, distribution, new to Thailand).

Distribution: China (Guangxi, Hainan, Hunan, Taiwan), Indonesia, Laos, Malaysia, Nepal, Thailand, Vietnam. 
2. Cestrotus heteropterus Shi et al. (2009, p.54). TL: China, Yunnan, Mengla, Lushilin.

Vietnamese Records:

Cestrotus heteropterus; Shi et al. (2011, p.673, distribution, new to Malaysia and Vietnam).

Distribution: China (Yunnan), Malaysia, Thailand, Vietnam.

3. Cestrotus quadrimaculatus Shi et al. (2011, p.668). TL: Thailand, Kamphaeng Phet, Khlong Lan, nr Khlong Lan waterfall.

Vietnamese Records:

Cestrotus quadrimaculatus; Shi et al. (2011, p.668, diagnosis, head and thorax photo, male genitalic figure, remarks).

Distribution: Thailand, Vietnam.

\section{Genus Dioides Kertesz, 1915}

TS: Dioides pictipennis Kertesz, 1915.

4. Dioides furcatus Shi, Li and Yang (2009, p.95). TL: China, Hainan, Yinggeling National Natural Reserve, Hongmao village.

Vietnamese Records:

Dioides furcatus; Shi et al. (2011, p.681, distribution, new to Thailand and Vietnam).

Distribution: China (Hainan), Thailand, Vietnam.

5. Dioides merzi Shi et al. (2011, p.674). TL: Vietnam, Tay Ninh, Nui Ba Den.

Vietnamese Records: 
Dioides merzi; Shi et al. (2011, p.674, diagnosis, distribution, male genitalic figure, remarks, wing photo).

Distribution: Thailand, Vietnam.

\section{Genus Homoneura Wulp, 1891}

TS: Homoneura (Homoneura) picea Wulp, 1891.

6. Homoneura (Chaetohomoneura) semibrunnea (Meijere, 1916, p.91 (Lauxania)). TL: Indonesia, Sumatra, Air Tarbis.

Vietnamese Records:

Homoneura (Chaetohomoneura) semibrunnea; Sasakawa (2001, p.56, description, distribution, new to Vietnam); Shi, Wang and Yang, 2011: 27 (distribution).

Distribution: Indonesia, Malaysia, Vietnam.

7. Homoneura (Homoneura) affinis Malloch (1929, p.81). TL: Philippines, Negros, Cuernos Mts.

Vietnamese Records:

Homoneura (Homoneura) affinis; Sasakawa (2001, pp.75, 79, in Vietnamese key, distribution, new to Vietnam); Sasakawa (2009, p.47, description, distribution, new to Micronesia).

Distribution: India, Indonesia, Malaysia, Micronesia, Philippines, Singapore, Russian Far East, Vietnam.

8. Homoneura (Homoneura) conspicua Sasakawa (2001, p.79). TL: Vietnam, Karyu Danar.

Vietnamese Records:

Homoneura (Homoneura) conspicua; Sasakawa (2001, pp.75, 79, in Vietnamese 
key, discussion, male genitalic figure).

Distribution: Vietnam.

9. Homoneura (Homoneura) cornuta Sasakawa (2001, p.85). TL: Vietnam, Vinh Phuc, Tam Dao.

Vietnamese Records:

Homoneura (Homoneura) cornuta; Sasakawa (2001, pp.76, 85, in Vietnamese key, diagnosis, discussion, male genitalic figure); Gao and Yang (2004, p.355, diagnosis, distribution, new to China, redescription, wing and male genitalic figure); Shi and Yang (2009b, p.470, distribution).

Distribution: China (Guangxi), Vietnam.

10. Homoneura (Homoneura) demeijerei Malloch (1929, p.75). TL: Indonesia, Sumatra, Bukittinggi.

Vietnamese Records:

Homoneura (Homoneura) demeijerei; Sasakawa (2001, pp.75, 78, in Vietnamese key, distribution, new to Vietnam); Lee et al. (2016, p.38, description, habitus photo).

Distribution: Indonesia, Malaysia, Vietnam.

11. Homoneura (Homoneura) discoalbata Sasakawa (1992, p.171). TL: Malaysia, Selangor, Kepong, FRIM.

Vietnamese Records:

Homoneura (Homoneura) discoalbata; Sasakawa (2001, pp.75, 85, in Vietnamese key, description, distribution, new to Vietnam).

Distribution: Malaysia, Vietnam.

12. Homoneura (Homoneura) discoglauca (Walker, 1860, p.147 (Ochthiphila)). 
TL: Indonesia, Sulawesi.

Vietnamese Records:

Homoneura (Homoneura) discoglauca; Sasakawa (2001, pp.76, 93, in Vietnamese key, distribution, new to Vietnam); Sasakawa (2009, p.49, description, distribution, new to Micronesia).

Distribution: Australia, China (Taiwan), Indonesia, Japan, Malaysia, Micronesia, Solomon Islands, Vietnam.

13. Homoneura (Homoneura) fasciventris Malloch (1927, p.169). TL: Taiwan.

Vietnamese Records:

Homoneura (Homoneura) fasciventris; Sasakawa (2001, pp.76, 94, in Vietnamese key, distribution, new to Vietnam); Sasakawa (2002, p.55, distribution); Sasakawa (2003, p.68, distribution, new to Laos); Shi and Yang (2009c, p.28, distribution); Li and Yang (2012, p.28, distribution).

Distribution: China (Taiwan), Indonesia, Laos, Malaysia, Vietnam.

14. Homoneura (Homoneura) grahami Malloch (1929, p.81). TL: China, Sichuan, Mt. Omei.

Vietnamese Records:

Homoneura (Homoneura) grahami; Sasakawa (2001, pp.75, 82, in Vietnamese key, description, distribution, new to Vietnam).

Distribution: China (Sichuan), Vietnam.

15. Homoneura (Homoneura) grandis (Kertesz, 1915, p.529 (Lauxania)). TL: Taiwan, Taihorinsho.

Vietnamese Records:

Homoneura (Homoneura) grandis; Sasakawa (2001, pp.76, 86, in Vietnamese 
key, distribution, new to Vietnam).

Distribution: China (Taiwan), Vietnam.

16. Homoneura (Homoneura) immaculata (Meujere, 1910, p.123 (Lauxania)). TL: Indonesia, Java, Wonosobo.

Vietnamese Records:

Homoneura (Homoneura) immaculata; Sasakawa (2001, pp.76, 94, in Vietnamese key, description, distribution, new to Vietnam); Sasakawa (2003, p.70, distribution, new to Laos).

Distribution: China (Guangxi, Guizhou, Hainan), Indonesia, Laos, Malaysia, Vietnam.

17. Homoneura (Homoneura) interrupta Sasakawa (2001, p.93). TL: Vietnam, Vinh Phuc, Tam Dao.

Vietnamese Records:

Homoneura (Homoneura) interrupta; Sasakawa (2001, pp.76, 93, in Vietnamese key, diagnosis).

Distribution: Vietnam.

18. Homoneura (Homoneura) laticosta (Thomson, 1869, p.598 (Geomyza)). TL: Singapore.

Vietnamese Records:

Homoneura (Homoneura) laticosta; Sasakawa (2001, pp.75, 82, in Vietnamese key, distribution, new to Vietnam); Sasakawa (2003, p.70, distribution, new to Laos); Sasakawa (2009, p.50, description, distribution, new to Micronesia).

Distribution: Australia, China (Taiwan), Indonesia, Laos, Malaysia, Micronesia, Philippines, Singapore, Solomon Islands, Thailand, Vietnam. 
19. Homoneura (Homoneura) lucida (Meijere, 1910, p.132 (Lauxania)). TL: Indonesia, Java, Djakarta.

Vietnamese Records:

Homoneura (Homoneura) lucida; Sasakawa (2001, pp.75, 82, in Vietnamese key, distribution, new to Vietnam).

Distribution: India, Indonesia, Malaysia, Philippines, Sri Lanka, Vietnam.

20. Homoneura (Homoneura) nubecula Sasakawa (2001, p.88). TL: Vietnam, Lao Cai, Sa Pa.

Vietnamese Records:

Homoneura (Homoneura) nubecula; Sasakawa (2001, pp.76, 88, in Vietnamese key, discussion, male genitalic figure).

Distribution: Vietnam.

21. Homoneura (Homoneura) ornatifrons (Kertesz, 1913, p.91 (Lauxania (Minettia))). TL: Taiwan, Kosempo.

Vietnamese Records:

Homoneura (Homoneura) ornatifrons; Sasakawa (2001, pp.76, 93, in Vietnamese key, description, distribution, new to Vietnam); Sasakawa (2002, p.57, distribution).

Distribution: Japan (Ryukyus), China (Taiwan), Vietnam.

22. Homoneura (Homoneura) parvibifida Sasakawa (2001, p.78). TL: Vietnam, Vinh Phuc, Tam Dao.

Vietnamese Records:

Homoneura (Homoneura) parvibifida; Sasakawa (2001, pp.75, 78, in Vietnamese key, discussion, male genitalic figure). 
Distribution: Vietnam.

23. Homoneura (Homoneura) philippinensis Malloch (1929, p.68). TL: Philippines.

Vietnamese Records:

Homoneura (Homoneura) philippinensis; Sasakawa (2001, pp.75, 77, in Vietnamese key, description, distribution, new to Vietnam).

Distribution: Malaysia, Philippines, Vietnam.

24. Homoneura (Homoneura) picta (Meijere, 1904, p.114 (Drosomyia)). TL: Indonesia, Java, Tosari.

Vietnamese Records:

Homoneura (Homoneura) picta; Sasakawa (2001, pp.76, 90, in Vietnamese key, distribution, new to Vietnam); Gao \& Yang (2004, p.361, diagnosis, distribution); Lee et al. (2016, p.39, description, habitus photo).

Distribution: China (Guangxi, Guizhou, Zhejiang, Taiwan), India, Indonesia, Malaysia, Nepal, Singapore, Thailand, Vietnam.

25. Homoneura (Homoneura) piliseta Malloch (1929, p.45). TL: not given.

Vietnamese Records:

Homoneura (Homoneura) piliseta; Sasakawa (2001, pp.76, 90, in Vietnamese key, description, distribution, new to Vietnam).

Distribution: Indonesia, Vietnam.

26. Homoneura (Homoneura) protuberans Sasakawa (2001, p.91). TL: Vietnam, Lam Dong, Dalat.

Vietnamese Records: 
Homoneura (Homoneura) protuberans; Sasakawa (2001, pp.76, 91, in Vietnamese key, diagnosis, discussion, male genitalic figure).

Distribution: Vietnam.

27. Homoneura (Homoneura) quiquenotata (Meijere, 1915, p.137 (Lauxania)). TL: Indoneia, Java.

Vietnamese Records:

Homoneura (Homoneura) quiquenotata; Sasakawa (2001, pp.76, 85, in Vietnamese key, distribution, new to Vietnam); Sasakawa (2003, p.71, distribution, new to Laos).

Distribution: China (Taiwan), Indonesia, Laos, Malaysia, Vietnam.

28. Homoneura (Homoneura) sauteri Malloch (1927, p.171). TL: Taiwan, Kosempo.

Vietnamese Records:

Homoneura (Homoneura) sauteri; Sasakawa (2001, pp.75, 81, in Vietnamese key, distribution, new to Vietnam); Sasakawa (2003, p.71, distribution, new to Laos); Shi and Yang (2009b, p.470, distribution).

Distribution: China (Hainan, Taiwan), Indonesia, Laos, Malaysia, Sri Lanka, Vietnam.

29. Homoneura (Homoneura) serristylis Sasakawa (2001, p.81). TL: Vietnam, Dak Lak, Buon Me Thuot.

Vietnamese Records:

Homoneura (Homoneura) serristylis; Sasakawa (2001, pp.75, 81, in Vietnamese key, male genitalic figure).

Distribution: Vietnam. 
30. Homoneura (Homoneura) signata (Wulp, 1881, p.52 (Minettia)). TL: Indonesia, Sumatra, Kloempang.

Vietnamese Records:

Homoneura (Homoneura) signata; Sasakawa (2001, pp.75, 81, in Vietnamese key, distribution, new to Cambodia and Vietnam); Sasakawa (2003, p.72, distribution, new to Laos); Shi and Yang (2009b, p.470, distribution).

Distribution: Cambodia, China (Heilongjiang), India, Indonesia, Laos, Malaysia, Philippines, Singapore, Thailand, Vietnam.

31. Homoneura (Homoneura) signatifrons (Kertesz, 1900, p.264 (Sapromyza)). TL: Papua New Guinea, West Sepik, Aitape, Seleo.

Vietnamese Records:

Homoneura (Homoneura) signatifrons; Sasakawa (2001, pp.76, 92, in Vietnamese key, description, distribution, new to Vietnam, male genitalic figure); Sasakawa (2009, p.54, description, distribution).

Distribution: Indonesia, Malaysia, Micronesia, Papua New Guinea, Solomon Islands, Vietnam.

32. Homoneura (Homoneura) striatifrons (Meijere, 1924, p.52 (Lauxania)). TL: Indonesia, Java, Tijbodas.

Vietnamese Records:

Homoneura (Homoneura) striatifrons; Sasakawa (2001, pp.75, 82, in Vietnamese key, distribution, new to Vietnam); Sasakawa (2003, p.72, distribution, new to Laos and Thailand).

Distribution: China (Taiwan), Indonesia, Japan (Ryukyus), Laos, Thailand, Vietnam.

33. Homoneura (Homoneura) strigata (Meijere, 1910, p.136 (Lauxania)). TL: 
Indonesia, Java.

Vietnamese Records:

Homoneura (Homoneura) strigata; Sasakawa (2001, pp.75, 84, in Vietnamese key, distribution, new to Vietnam); Sasakawa (2003, p.72, distribution, new to Laos); Lee et al. (2016, p.40, description, habitus photo).

Distribution: Indonesia, Laos, Malaysia, Vietnam.

34. Homoneura (Homoneura) subnubecula Sasakawa (2001, p.89). TL: Vietnam, Lao Cai, Sa Pa.

Vietnamese Records:

Homoneura (Homoneura) subnubecula; Sasakawa (2001, pp.76, 89, in Vietnamese key, discussion, male genitalic figure).

Distribution: Vietnam.

35. Homoneura (Homoneura) trivittata Sasakawa (1992, p.205). TL: Malaysia, Borneo, Sabah, Sepilok Forest Reserve.

Vietnamese Records:

Homoneura (Homoneura) trivittata; Sasakawa (2001, pp.76, 85, in Vietnamese key, distribution, new to Vietnam).

Distribution: Indonesia, Malaysia, Vietnam.

36. Homoneura (Homoneura) trypetoptera (Hendel, 1908, p.27 (Lauxania (Sapromyza))). TL: Vietnam.

Vietnamese Records:

Homoneura (Homoneura) trypetoptera; Sasakawa (1992, p.207, description, distribution, new to Malaysia and Borneo, male genitalic figure); Sasakawa (1998, p.57, distribution); Sasakawa (2001, pp.76, 90, in Vietnamese key, distribution); Sasakawa 
(2003, p.74, distribution, new to Laos); Lee et al. (2016, p.41, description, habitus photo).

Distribution: China (Taiwan), India, Indonesia, Laos, Malaysia, Nepal, Philippines, Sri Lanka, Thailand, Vietnam.

37. Homoneura (Homoneura) undulata Sasakawa (2001, p.76). TL: Vietnam, Khanh Hoa, Ninh Hoa, North of Nha Trang.

Vietnamese Records:

Homoneura (Homoneura) undulata; Sasakawa (2001, pp.75, 76, in Vietnamese key, discussion, male genitalic figure).

Distribution: Vietnam.

38. Homoneura (Homoneura) unguiculata (Kertesz, 1913, p.100 (Lauxania (Minettia))). TL: Taiwan, Takao, Yentempo, Koshun, Tainan, Taihoku.

Vietnamese Records:

Homoneura (Homoneura) unguiculata; Shatalkin (1996, p.182, in Palaearctic key, distribution); Shatalkin (2000, p.29, in Palaearctic key, distribution); Sasakawa (2001, pp.75, 79, in Vietnamese key, distribution, new to Vietnam); Sasakawa (2002, p.58, distribution); Schacht, Kurina, Merz and Gaimari (2004, p.54, English translation of Shatalkin, 2000, p.29).

Distribution: China (Taiwan), Japan, North Korea, Sri Lanka, USA (immigrant), Vietnam.

39. Homoneura (Homoneura) vietnamensis Sasakawa (2001, p.86). TL: Vietnam, Lao Cai, Sa Pa.

Vietnamese Records:

Homoneura (Homoneura) vietnamensis; Sasakawa (2001, pp.76, 86, in Vietnamese key, discussion, male ginitalic figure). 
Distribution: Vietnam.

40. Homoneura (Homoneura) vittigera Sasakawa (2001, p.82). TL: Vietnam, Vinh Phuc, Tam Dao.

Vietnamese Records:

Homoneura (Homoneura) vittigera; Sasakawa (2001, pp.75, 82, in Vietnamese key, discussion, male genitalic figure).

Distribution: China (Yunnan), Vietnam.

41. Homoneura (Minettioides) forcipata (Kertesz, 1913, p.100 (Lauxania (Minettia))). TL: Taiwan, Takao.

Vietnamese Records:

Homoneura (Homoneura) forcipata; Sasakawa (2001, pp.75, 81, in Vietnamese key, distribution, new to Vietnam).

Homoneura (Minettioides) forcipata; Shi, Gaimari and Yang (2012, p.6, diagnosis, distribution).

Distribution: China (Taiwan), Malaysia, Micronesia, Vietnam.

42. Homoneura (Neohomoneura) apodemata Sasakawa (2001, p.61). TL: Vietnam, Lam Dong, Fyan.

Vietnamese Records:

Homoneura (Neohomoneura) apodemata; Sasakawa (2001, pp.57, 61, in Vietnamese key, discussion, male genitalic figure); Shi and Yang (2008, p.46, distribution); Shi et al. (2011, p.27, distribution).

Distribution: Vietnam.

43. Homoneura (Neohomoneura) bigibba Sasakawa (2001, p.70). TL: Vietnam, Lam Dong, Fyan. 
Vietnamese Records:

Homoneura (Neohomoneura) bigibba; Sasakawa (2001, pp.58, 70, in Vietnamese key, male genitalic figure, remarks); Sasakawa (2003, p.66, description, distribution, new to Laos); Shi and Yang (2008, p.46, distribution); Shi et al. (2011, p.27, distribution).

Distribution: Laos, Vietnam.

44. Homoneura (Neohomoneura) caudata Sasakawa (2001, p.59). TL: Vietnam, Lam Dong, Fyan.

Vietnamese Records:

Homoneura (Neohomoneura) caudata; Sasakawa (2001, pp.57, 59, in Vietnamese key, diagnosis, male genitalic figure); Shi and Yang (2008, p.46, distribution); Shi et al. (2011, p.27, distribution).

Distribution: Vietnam.

45. Homoneura (Neohomoneura) clavigera Sasakawa (2001, p.67). TL: Vietnam, Lam Dong, Fyan.

Vietnamese Records:

Homoneura (Neohomoneura) clavigera; Sasakawa (2001, pp.58, 67, in Vietnamese key, discussion, male genitalic figure); Shi and Yang (2008, p.46, distribution); Shi et al. (2011, p.27, distribution).

Distribution: Vietnam.

46. Homoneura (Neohomoneura) diphylla Sasakawa (2001, p.58). TL: Vietnam, Lam Dong, Di Linh.

Vietnamese Records:

Homoneura (Neohomoneura) diphylla; Sasakawa (2001, pp.57, 58, in 
Vietnamese key, diagnosis, male genitalic figure); Sasakawa (2003, p.66, distribution, new to Laos); Shi and Yang (2008, p.46, distribution); Shi et al. (2011, p.27, distribution).

Distribution: Laos, Vietnam.

47. Homoneura (Neohomoneura) dolabrifera Sasakawa (2001, p.65). TL: Vietnam, Vinh Phuc, Tam Dao.

Vietnamese Records:

Homoneura (Neohomoneura) dolabrifera; Sasakawa (2001, pp.57, 65, in Vietnamese key, discussion, male genitalic figure); Shi and Yang (2008, p.46, distribution); Shi et al. (2011, p.27, distribution).

Distribution: Vietnam.

48. Homoneura (Neohomoneura) honesta (Kertesz, 1915, p.532 (Lauxania (Minettia))). TL: Taiwan, Kosempo.

Vietnamese Records:

Homoneura (Neohomoneura) honesta; Sasakawa (2001, pp.58, 72, in Vietnamese key, distribution, new to Cambodia and Vietnam); Sasakawa (2002, p.61, description, distribution); Sasakawa (2003, p.67, distribution, new to Laos); Shi and Yang (2008, p.46, distribution); Shi et al. (2011, p.27, distribution).

Distribution: Cambodia, China (Taiwan), Laos, Malaysia, Thailand, Vietnam.

49. Homoneura (Neohomoneura) incompleta Malloch (1927, p.108). TL: Indonesia, Sumatra, Bukittinggi.

Vietnamese Records:

Homoneura (Neohomoneura) incompleta; Sasakawa (2001, pp.58, 74, in Vietnamese key, description, distribution, new to Vietnam, male genitalic figure); Shi and Yang (2008, p.46, distribution); Shi et al. (2011, p.27, distribution). 
Distribution: China (Guangxi, Hainan, Yunnan), Indonesia, Vietnam.

50. Homoneura (Neohomoneura) lateriloba Sasakawa (2001, p.67). TL: Vietnam, Lam Dong, Fyan.

Vietnamese Records:

Homoneura (Neohomoneura) lateriloba; Sasakawa (2001, pp.58, 67, in Vietnamese key, diagnosis, male genitalic figure); Sasakawa (2003, p.67, distribution, new to Laos); Shi and Yang (2008, p.46, distribution); Shi et al. (2011, p.27, distribution).

Distribution: Laos, Vietnam.

51. Homoneura (Neohomoneura) longiseta Sasakawa (2001, p.62). TL: Vietnam, Lam Dong, Fyan.

Vietnamese Records:

Homoneura (Neohomoneura) longiseta; Sasakawa (2001, pp.57, 62, in Vietnamese key, diagnosis, male genitalic figure, remarks); Sasakawa (2003, p.67, distribution, new to Laos); Shi and Yang (2008, p.46, distribution); Shi et al. (2011, p.27, distribution).

Distribution: Laos, Vietnam.

52. Homoneura (Neohomoneura) mediomaculata Sasakawa (2001, p.65). TL: Vietnam, Khanh Hoa, Dai Lanh.

Vietnamese Records:

Homoneura (Neohomoneura) mediomaculata; Sasakawa (2001, pp.57, 65, in Vietnamese key, discussion, male genitalic figure); Shi and Yang (2008, p.46, distribution); Shi et al. (2011, p.27, distribution).

Distribution: Cambodia, Vietnam. 
53. Homoneura (Neohomoneura) nebulosa Sasakawa (2001, p.72). TL: Vietnam, Vinh Phuc, Tam Dao.

Vietnamese Records:

Homoneura (Neohomoneura) nebulosa; Sasakawa (2001, pp.58, 72, in Vietnamese key, diagnosis, male genitalic figure); Shi and Yang (2008, p.46, distribution); Shi et al. (2011, p.19, description, diagnosis, distribution, remarks, wing photo).

Distribution: China (Guangxi), Vietnam.

54. Homoneura (Neohomoneura) nigronotata (Kertesz, 1915, p.530 (Lauxania)). TL: Taiwan.

Vietnamese Records:

Homoneura (Neohomoneura) nigronotata; Sasakawa (2001, p.57, in Vietnamese key); Shi and Yang (2008, p.46, distribution); Shi and Yang (2009b, p.470, distribution); Shi et al. (2011, p.27, distribution).

Distribution: China (Taiwan), Vietnam.

55. Homoneura (Neohomoneura) paroeca (Kertesz, 1915, p.531 (Lauxania)). TL: Taiwan, Kosempo.

Vietnamese Records:

Homoneura (Neohomoneura) paroeca; Sasakawa (2001, p.57, in Vietnamese key); Shi and Yang (2008, p.46, distribution); Shi et al. (2011, p.27, distribution).

Distribution: China (Taiwan), Vietnam.

56. Homoneura (Neohomoneura) pollex Sasakawa (2001, p.69). TL: Vietnam, Vinh Phuc, Tam Dao.

Vietnamese Records: 
Homoneura (Neohomoneura) pollex; Sasakawa (2001, pp.58, 69, in Vietnamese key, discussion, male genitalic figure, remarks); Shi and Yang (2008, pp.35, 46, diagnosis, distribution, new to China); Shi et al. (2011, p.27, distribution).

Distribution: China (Taiwan), Vietnam.

57. Homoneura (Neohomoneura) subhonesta Sasakawa (2001, p.71). TL: Vietnam, Lam Dong, Fyan.

Vietnamese Records:

Homoneura (Neohomoneura) subhonesta; Sasakawa (2001, pp.58, 71, in Vietnamese key, discussion, male genitalic figure); Shi and Yang (2008, p.46, distribution); Shi et al. (2011, p.28, distribution).

Distribution: Vietnam.

58. Homoneura (Neohomoneura) turbida Sasakawa (2001, p.63). TL: Vietnam, Lam Dong, Fyan.

Vietnamese Records:

Homoneura (Neohomoneura) turbida; Sasakawa (2001, pp.57, 63, in Vietnamese key, discussion, male genitalic figure); Shi and Yang (2008, 46, distribution); Shi et al. (2011, p.28, distribution).

Distribution: Vietnam.

\section{Genus Phobeticomyia Kertesz, 1915}

TS: Phobeticomyia lunifera (Meijere, 1910).

59. Phobeticomyia lunifera (Meijere, 1910, p.134 (Lauxania)). TL: Indonesia, Java, Jakarta.

Vietnamese Records:

Phobeticomyia lunifera; Sasakawa (2001, p.55, distribution, new to Vietnam); 
Shi, Li and Yang (2009b, p.61, diagnosis, distribution, head and wing photo, male genitalic figure, remarks).

Distribution: China (Hainan, Taiwan), India, Indonesia, Malaysia, Nepal, Philippines, Sri Lanka, Thailand, Vietnam.

\section{Genus Prosopophorella Meijere, 1918}

TS: Prosopophorella buccata (Meijere, 1910).

60. Prosopophorella yoshiyasui Sasakawa (2001, p.54). TL: Vietnam, Vinh Phuc, Tam Dao.

Vietnamese Records:

Prosopophorella yoshiyasui; Sasakawa (2001, p.54, discussion, head and wing figure); Shi and Yang (2009a, p.160, description, diagnosis, distribution, new to China, head and wing photo, male genitalic figure).

Distribution: China (Guangxi), Vietnam.

\section{ACKNOWLEDGEMENTS}

This work was supported by a grant from the National Institute of Biological Resources (NIBR), funded by the Ministry of Environment of (MOE) of the Republic of Korea (NIBR201604201). I am grateful to the members of Biology Faculty, Dalat University for providing an intellectually inspiring environment for this study.

\section{REFERENCES}

Gao, C., \& Yang, D. (2004). A Review of the genus Homoneura from Guangxi, China (Diptera: Lauxaniidae). Raffles Bulletin of Zoology, 52(2), 351-364.

Hendel, F. (1907). Neue und interessante Dipteren aus dem keiserl Museum in Wien. (Ein Beitrag zur Kenntnis der acalyptraten Musciden.). Wiener Entomologische Zeitung, 26, 223-245.

Hendel, F. (1908). Diptera. Fam. Muscaridae. Subfam. Lauxaninae. Genera Insectorum, $68,1-66$. 
Kertesz, K. (1900). Beitrage zur Kenntniss der Indo-Australischen. Sapromyza-Arten. Termesz. Fuz., 23, 254-275.

Kertesz, K. (1913). H. Sauter's Formosa-Ausbeute. Lauxaniidae. (Dipt.). Ann. Hist.-Nat. Mus. Natl. Hung, 11, 88-102.

Kertesz, K. (1915). H. Sauter's Formosa-Ausbeute. Lauxaniidae. II. Annls hist.-nat. Mus. Natn. Hung, 13, 491-534.

Kim, S. P. (1994). Revision of the Australian species of Homoneura vad der Wulp, Trypetisoma Malloch, and allied genera (Diptera: Lauxaniidae). Monographs on invertebrate taxonomy (Vol. 1). Collingwood, Australia: CSIRO Publications Australia.

Lee, H. S., Bae, Y. S., Ram, K. D., Lee, J. W., Choi, J. K., Kim, Y. H., Choi, D. S., Byun, H. W., Jeon, M. J., \& Nguyen, V. K. (2016). Biodiversity of Southern Vietnam - Insects. Incheon, Korea: National Institute of Biological Resources.

Li, W., \& Yang, D. (2012). Eleven new species of the Homoneura (Homoneura) beckeri group from Yunnan, China (Diptera, Lauxaniidae). Zootaxa, 3537, 1-28.

Loew, H. (1862). Bidrag till Kannedomen om Afrikas Diptera. Ofvers $K$. Vetenskapsakad. Forth, 19, 3-14.

Malloch, J. R. (1927). Fauna Sumatrensis (Beitrag Nr. 40). Sapromyzidae (Dipt.). Suppl. Ent., 15, 102-110.

Malloch, J. R. (1927). H. Sauter's Formosa collection: Sapromyzidae (Dipt.). Ent. Mitt., $16,159-172$.

Malloch, J. R. (1929). Notes on some oriental sapromyzid flies (Diptera), with particular reference to the Philippines species. Proc. U.S. Natl. Mus. 74(6), 1-97.

Meijere, J. C. H. de (1904). Neue und bekannte Sud-Asiatische Dipteren. Bijdr. Dierkd., $18,85-117$.

Meijere, J. C. H. de (1910). Studien uber sudostasiatische Dipteren. IV. Die neue Dipterenfauna von Krakatau. Tijdschr. Ent. 53, 58-194.

Meijere, J. C. H. de (1915). Diptera aus Nord-Neu-Guinea gesammelt von Dr. P. N. van Kampen und K. Gjellerup in den Jahren 1910 und 1911. Tijdschr. Entomol., 58, 98-139.

Meijere, J. C. H. de (1916). Studien uber sudostasiatische Dipteren X. Dipteren von Sumatra. Tijdschr. Ent., 58 (Suppl.), 64-97.

Meijere, J. C. H. de (1918). Studien uber sudostasiatische Dipteren XIV. Verzeichnis der von mir behandelten Arten. Tijdschr. Ent., 60, 275-369.

Meijere, J. C. H. de (1924). Studien uber sudostasiatische Dipteren XV. Dritter Beitraz zur Kenntnis der sumatranischen Dipteren. Tijdschr. Ent., 67 (Suppl.), 1-64.

Meijere, J. C. H. de (1924). Studien uber sudostasiatische Dipteren XVI. Tijdschr. Ent., 67, 197-224.

Sasakawa, M. (1992). Lauxaniidae (Diptera) of Malaysia (part 2): A revision of 
Homoneura van der Wulp. Insecta Matsumurana, 46, 133-210.

Sasakawa, M. (1998). Oriental Lauxaniidae (Diptera) Part 1. Sci. Rep. Kyoto Pref. Univ., Hum. Env. \& Agr., 50, 49-74.

Sasakawa, M. (2001). Oriental Lauxaniidae (Diptera) part 2. Fauna of the Lauxaniidae of Viet Nam. Sci. Rep. Kyoto Pref. Univ., Hum. Env. \& Agr., 53, 39-94.

Sasakawa, M. (2002). Oriental Lauxaniidae (Diptera) part 3. Fauna of the Lauxaniidae in Japan (Ryukyus) and Formosa. Sci. Rep. Kyoto Pref. Univ., Hum. Env. \& Agr., 54, 33-61.

Sasakawa, M. (2003). Oriental Lauxaniidae (Diptera) part 4. Fauna of the Lauxaniidae of Laos. Sci. Rep. Kyoto Pref. Univ., Hum. Env. \& Agr., 55, 57-74.

Sasakawa, M. (2009). Insects of Micronesia, Diptera: Lauxaniidae. Micronesica, 41(1), 33-57.

Schacht, W., Kurina, O., Merz, B., \& Gaimari, S. D. (2004). Zweiflugler aus Bayern XXII (Diptera: Lauxaniidae, Chamaemyiidae). Entomofauna Zeitschrift Fur Entomologie, 25, 41-80.

Shatalkin, A. I. (1996). Palearctic Species of Homoneura (Diptera, Lauxaniidae). Entomological Review, 75(7), 171-186.

Shatalkin, A. I. (2000). Keys to the Palaearctic flies of the family Lauxaniidae (Diptera). Zoologicheskie Issledovania, 5, 1-102.

Shewell, G. E. (1977). Family Lauxaniidae. Cat. Dipt. Orient. Reg., 3. 182-214.

Shi, L., \& Yang, D. (2008). Species of the subgenus Neohomoneura van der Wulp from Hainan in China (Diptera, Lauxaniidae). Zootaxa, 1793, 28-46.

Shi, L., \& Yang, D. (2009a). Species of the Genus Prosopophorella from China (Diptera: Lauxaniidae). Annales Zoologici, 59(2), 159-164.

Shi, L., \& Yang, D. (2009b). Notes on species groups of subgenus Homoneura from China with descriptions of two new species (Diptera, Lauxaniidae). Acta Zootaxonomica Sinica, 34(3), 462-471.

Shi, L., \& Yang, D. (2009c). Notes on Homoneura (Homoneura) beckeri group from the Oriental Region, with descriptions of ten new species from China (Diptera: Lauxaniidae). Zootaxa, 2325, 1-28.

Shi, L., Yang, D., \& Gaimari, S. D. (2009). Species of the genus Cestrotus Loew from China (Diptera: Lauxaniidae). Zootaxa, 2090, 41-68.

Shi, L., Li, W. L., \& Yang, D. (2009a). Five new species of the genus Dioides from China (Diptera: Lauxaniidae). Annales Zoologici, 59(1), 93-105.

Shi, L., Li, W., \& Yang, D. (2009b). Two new species of the genus Phobeticomyia from China (Diptera, Lauxaniidae). Zootaxa, 2090, 57-68.

Shi, L., Yang, D., \& Gaimari, S. D. (2011). Four new species from China and Southeast Asia (Diptera, Lauxaniidae, Homoneurinae). Revue Suisse de Zoologie. 118(4), 667-693. 
Shi, L., Wang, J. C., \& Yang, D. (2011). Nine new species from China of the subgenera Chaetohomoneura and Neohomoneura in the genus Homoneura (Diptera, Lauxaniidae). Zootaxa, 2975, 1-28.

Shi, L., Gaimari, S. D., \& Yang, D. (2012). Notes on the Homoneura subgenera Euhomoneura, Homoneura and Minettioides from China (Diptera: Lauxaniidae). Zootaxa, 3238, 1-22.

Thomson, C. G. (1869). Diptera. Species nova descripsit. In C. A. Virgin, Kongliga svenska fregatten Eugenies resa omkring Jordan under befäll af C. A. Virgin, aren 1851-1853. Vol. 2. (pp. 443-614). Stockholm: P. A. Norstedt \& Söner.

Walker, F. (1860). Catalogue of the dipterous insects collected at Makessar in Celebes, by Mr. A. R. Wallace, with descriptions of new species. J. Proc. Linn. Soc. London Zool., 4, 145-172.

Wulp, F. M. van der. (1881). Natuurlijke Histoire. Negende afdeeling. Diptera. In P. J. Veth, Midden-Sumatra. Reizen en onderzoekingen der Sumatra-expeditie, uitgerust door het Aardrijkskundig genootschap, 1877-1879, beschreven door de leden der expeditie, onder toezicht van Prof. P. J. Veth (pp. 1-60). E. J. Brill, Leiden.

Wulp, F. M. van der. (1891). Eenige uitlandsche Diptera. Tijdschr. Ent. (1890-1891), 34, 193-218.

\title{
DANH LỤC CÁC BậC PHÂN LOẠI THUỘC HỌ LAUXANIIDAE (DIPTERA, LAUXANIOIDEA) Ở VIẸT NAM
}

\author{
Hyun Suk Lee ${ }^{\mathrm{a} *}$ \\ ${ }^{a}$ Khoa Sinh họ, Trường Đại học Đà Lạt, Lâm Đồng, Việt Nam \\ *Tác giả liên hệ: Email: exuknight@gmail.com \\ Lịch sử bài báo \\ Nhận ngày 10 tháng 08 năm 2016 | Chỉnh sửa ngày 20 tháng 09 năm 2016 \\ Chấp nhận đăng ngày 30 tháng 09 năm 2016
}

\begin{abstract}
Dựa kết quả thu thập mẫu vật Lauxaniid và các tài liệu, danh lục thành phần các bậc phân loại thuộc Lauxaniid ở Việt Nam đã được thống kê. Kết quả nghiên cúu, Lauxaniid thuộc 2 phân họ, 60 loài. Tuy nhiên đây là kết quả bước đầu, cần phải tiến hành nghiên cứu kỹ hơn nữa dụa trên mẫu vật thu được một cách toàn diện.
\end{abstract}

Từ khóa: Diptera; Danh lục; Homoneurinae; Lauxaniidae; Việt Nam. 